\title{
Three-Electron Dynamics of the Interparticle Coulombic Decay with
} Two-Dimensional Continuum Confinement

\author{
Fabian Langkabel ${ }^{1, a)}$ and Annika Bande ${ }^{1, b}$ ) \\ Helmholtz-Zentrum Berlin für Materialien und Energie GmbH, Hahn-Meitner-Platz 1, \\ 14109 Berlin, Germany
}

(Dated: 15 January 2021)

In a pair of self-assembled or gated laterally-arranged quantum dots, an electronically excited state can undergo interparticle Coulombic decay. Then an electron from a neighbor quantum dot is emitted into the electronic continuum along the two available dimensions. This study proves that the process is not only operative among two, but also among three quantum dots, where a second electron-emitting dot causes a rate increase by a factor of two according to the predictions from the analytical Wigner-Weisskopf rate equation. The predictions hold over the complete range of conformation angles among the quantum dots and over a large range of distances. Electron dynamics was calculated by multiconfiguration time-dependent Hartree and is, irrespective of the large number of discrete variable representation grid points, feasible after having developed an OpenACC graphic card compilation of the program.

\footnotetext{
a) Also at the Institute of Chemistry and Biochemistry, Freie Universität Berlin, Arnimallee 22, 14195 Berlin, Germany; Formerly at the Department of Chemistry, Humboldt-Universität zu Berlin, Brook-Taylor-Str. 2, 12489 Berlin, Germany

${ }^{b)}$ Electronic mail: annika.bande@ @elmholtz-berlin.de; https://hz-b.de/theochem
} 


\section{INTRODUCTION}

For more than two decades the interparticle Coulombic decay (ICD) process created a stir. ${ }^{1-7}$ Theoreticians and experimentalist from disciplines ranging from atomic cluster physics ${ }^{8-17}$ over biomedicine $^{18,19}$ to nanophysics ${ }^{20-22}$ traced ICD and found it being effective in the various systems. This general presence originates probably from the astonishing simplicity of the process. Nothing more happens than that an inner-valence excited or ionized electronic structure relaxes into its ground state efficiently, because a neighboring structure has a valence electron that can take up the excess energy via Coulomb interaction only and is ionized in response. Many studies in the experimental disciplines were governed by energetic and structural considerations, i.e. one would know which Coulomb explosion products are collected in ion and electron detectors and what energy those have and reconstruct from this the progression of ICD. ${ }^{8,9,17}$ Or one would record photoelectron spectra. ${ }^{23-25}$ A recent alternative are pump-probe techniques. ${ }^{16,26}$ The vast majority of theories considers initial resonances, final ionized states, and Coulomb interaction integrals, which is challenging, as highly-excited and continuum states are to be calculated accurately. 2,4 Eventually, nuclear dynamics calculations aid to simulate the Coulomb explosion and deliver kinetic energy releases. ${ }^{10,27-31}$ They were recently combined with electron dynamics. ${ }^{32}$

And indeed, as ICD is an ultrafast electronic decay process, it is of high interest to investigate it in the short-time domain of electron dynamics. This holds two interconnected challenges. One is the number of electrons, which, for reasons of computational feasibility, can only be in the order of ten, at least if highly-correlated wavefunction-based methods are used. The other is electron correlation itself, which is essential to an accurate computation of the Coulomb interaction that is mediating ICD. Thus, in using the multiconfiguration time-dependent Hartree method (MCTDH) ${ }^{33,34}$ with antisymmetrization, a body of research works on the transient nature of ICD was formed to benchmark the fundamentals of ICD. Gaussian binding potentials were used, which represent the essentials of the electronic structure, of atoms or molecules in that they reflect a quasi-atomic structure. Moreover, they are established models for quantum dots (QDs).

The gains drawn from elucidating the electron dynamics during ICD so far are the following. First, the decays of two-site triplet ${ }^{35}$ or singlet $^{36}$ ICD resonance states were shown in an effectively one-dimensional model, where the ICD electron can only leave along the coordinate connecting the two binding potentials, as was found likewise in the case of quantum wells. ${ }^{37,38}$ The frequently considered analytical and approximate rate equation for well-separated subsystems ${ }^{39,40}$ was con- 
firmed in a separation-dependence study, showing that the Coulomb interaction of two neighbors alone determines the rate. But it revealed also that a one-dimensional continuum is a special case in which rates are subject to oscillations around the monotonic behavior predicted from the asymptotic formulae. Second, the relative energy of electronic levels, modified in QDs through geometries, was shown to affect the ICD rate partially in a systematic, ${ }^{41}$ but partially also in a non-trivial way. ${ }^{42}$ Third, it was shown that both electron capture ${ }^{43,44}$ and electromagnetic oscillating fields ${ }^{45,46}$ can initiate ICD. Fourth, the decay is altered by level atom or potential vibrations. ${ }^{47}$ Fifth, the role of an additional potential was elucidated from three viewing angles. When being at a long distance and only shallow or even repulsive, which means the extra potential can be interpreted as an impurity in the QD framework, then a non-trivial alternation of the ICD rates is discovered. ${ }^{48}$ When being a somewhat deeper binding potential in short distance from the two partners undergoing ICD, the extra site, a QD of other geometry or material composition, supports superexchange ICD. ${ }^{49}$ And when a full-depth binding potential is added, i.e. another QD of the same material as the others, it acts as a participator in a three-electron ICD process. ${ }^{50}$ This means among others that it supports the prefactor for the number of neighbors $N$ predicted from the aforementioned analytical rate equation. 39,40

Just recently, the ICD rate equation was also confirmed in electron dynamics calculation in two-electron systems with a two-dimensional electronic continuum. ${ }^{48,51}$ The respective potentials correspond to laterally-arranged self-assembled ${ }^{52-54}$ or electrostatically confined QDs. ${ }^{55}$ There, the dynamics rates showed much less pronounced oscillations with parameter variations in comparison to the analytical monotonic rate as function of the respective parameter, where the parameter considered so far was the inter-QD distance. In the past years it was found that the related electron-transfer mediated decay process of three atoms depends on their exact constellation. ${ }^{56} \mathrm{We}$ assume the same for ICD. Therefore, we consider in this study systems of three binding sites with a two-dimensional continuum and a two-dimensional conformation space for their arrangement.

For the realm of electron dynamics calculations in QD models, this attempt is a novelty, because it bridges among knowledge on three electrons in three sites ${ }^{50}$ and knowledge on a twodimensional continuum. ${ }^{48,51}$ We anticipate a reduction of rate fluctuations and an increase of the rate by a factor of two compared to the ICD rate with one neighbor only. The unknown regarding the physics is on the role of the spatial arrangement of the three potentials in a straight or angled row. Moreover, previous calculations with three electrons and more so with two continuum dimensions showed increases the computation time within the Heidelberg MCTDH program ${ }^{57,58}$ 
due to the required increase basis size in discrete variable representation (DVR). ${ }^{57,59,60}$ Therefore, an essential technical development accompanies this work, which is namely a compilation and parallelization of MCTDH on graphics processing units (GPUs).

After introducing the method, model, (Sec. II) and parametrization (Sec. III A), we hence introduce the GPU setup of MCTDH (Sec. III B). In the results we discuss the latter at first (Sec. IV A), before we reveal the electronic structure (Sec. IV B) and dynamics in the linear (Sec. IV C 1) or angled (Sec. IV C 2) arrangement of the three sites and lead all to a concise conclusion (Sec. V).

\section{THEORY}

The method utilized to investigate ICD of an electronically excited state of one QD in the neighborhood of two others QDs allowing for ionization is the multiconfiguration time-dependent Hartree method (MCTDH) ${ }^{33,34}$ in the Heidelberg implementation. ${ }^{57,58}$ With imposing appropriate antisymmetry constraints to the wavefunction, MCTDH allows to solve the electronic timedependent Schrödinger equation for the three-electrons-in-three-QDs problem. The method has proven successful for the investigation of three QDs in a nanowire. ${ }^{50}$ Here it is going to be extended for laterally arranged QDs with a two-dimensional continuum, for which a model has been developed in another work considering two electrons. ${ }^{48,51}$

The electronic Hamiltonian contains the typical operators of the kinetic energy, the QD binding potential, the Coulomb interaction, and the complex absorbing potential (from left to right) and reads

$$
\hat{H}=\sum_{i}\left(\hat{T}_{i}+\hat{V}_{i}^{Q D}+\sum_{j<i} \hat{r}_{i j}^{-1}+\mathrm{i} \hat{W}_{i}\right) .
$$

Here the indices $i, j=1,2,3$ run over the two or three electrons of the two comparative sets of calculations. The potential $\hat{V}_{i}^{Q D}$ reflects the two or three QDs from which electrons can be emitted into a two-dimensional unconfined (continuum) region. Two-dimensional Gaussians,

$$
\hat{V}_{i}^{Q D}\left(x_{i}, z_{i}\right)=\sum_{q}-D_{q} \cdot \exp \left\{-b_{q}\left[\left(\hat{z}_{i}-z_{q}\right)^{2}+\left(\hat{x}_{i}-x_{q}\right)^{2}\right]\right\}
$$

as function of $x_{i}$ and $z_{i}$ serve their description. $q$ refers to the light-absorbing (A) and the two identical electron-emitting (E, $\left.\mathrm{E}^{\prime}\right)$ QDs. The central QD is the light-absorbing one and accommodates the energy levels $A_{1}$ and $A_{0}$, where the higher is two-fold degenerate with respect to the two dimensions $x$ and $z$ if only the isolated AQD is considered. The isolated outer, electron-emitting 
QDs accommodate each only one level, $E_{0}$ and $E_{0}^{\prime}$. Note that in the two-QD case no $E^{\prime} \mathrm{QD}$ and no third electron is considered. The remaining variables of Eq. (2) are the QD positions $x_{q}$ and $z_{q}$ on the respective coordinates as well as the the potentials' depths $D_{q}$ and their widths parameters $b_{q}=4 \ln (2) / r_{q}^{2}$, connecting to the half widths at half maximum $r_{q}$. They are tuned such that $E_{A 1}>E_{E 0}>E_{A 0}$ and that the excitation energy in the AQD, $\omega_{A}=E_{A 1}-E_{A 0}$, exceeds the ionization energy $I P=-E_{E 0}$ to assure that ICD is energetically allowed. ${ }^{35}$

The Coulomb interaction among every two electrons, i.e. the essential mediator to ICD, is given in the approximate two-dimensional representation ${ }^{51}$

$$
\hat{r}_{i j}^{-1} \Rightarrow\left[\hat{r}_{i j}^{2}+\alpha^{2} \cdot e^{-\beta \hat{r}_{i j}}\right]^{-1 / 2} \text {. }
$$

$r_{i j}=\left|\mathbf{r}_{i}-\mathbf{r}_{j}\right| \rightarrow \sqrt{\left(x_{i}-x_{j}\right)^{2}+\left(z_{i}-z_{j}\right)^{2}}$ is the distance among electron $i$ and $j$. Furthermore, a regularization through $\alpha$ and $\beta$ reduces the Coulomb interaction to a finite value at the coincidence point of both electrons. This is necessary for the numerical stability within the MCTDH framework, in which a pseudo-spectral basis in form of a finite discrete variable representation (DVR) $)^{57,59,60}$ is used. Another important aspect is the required sum-of-products form of the fourdimensional interaction (Eq. (3)). The multi-grid POTFIT (MGPF) ${ }^{61}$ routine was proven to do this transformation in conjunction with a computationally inexpensive and sufficiently accurate truncation of the number of single-particle potential terms. ${ }^{51}$

$\hat{W}_{i}$ are the complex absorption potentials (CAPs) ${ }^{62-65}$ that apply to each electron. They also apply to each of the six spatial coordinates $c_{i}=x_{i}, z_{i}$ in positive and negative direction \pm . Therefore they are defined through

$$
\mathrm{i} \hat{W}_{i}=\mathrm{i} \sum_{c_{i}} \sum_{ \pm} \hat{W}_{c_{i}}^{ \pm}=\mathrm{i} \eta\left|c_{i}-c_{i}^{ \pm}\right|^{n} \Theta\left(c_{i}-c_{i}^{n, p}\right)
$$

The mathematical form of a CAP is a half-sided (Heavyside function $\Theta$ ) $n$-th order polynomial with onset $c_{i}^{ \pm}$. Through its strength $\eta$ it is tuned such that it optimally absorbs the continuum parts of the MCTDH wavefunction.

The core method is to solve the MCTDH equations of motion ${ }^{33,34}$ that arise when introducing the MCTDH wavefunction into the Dirac-Frenkel variational principle. This solves the timedependent electronic Schrödinger equation. The wave function is, in the whole series of papers on QD-ICD, here for the first time six-dimensional with coordinates $c_{1}, \ldots, c_{6}=x_{1}, x_{2}, x_{3}, z_{1}, z_{2}, z_{3}$ and reads

$$
\Psi\left(c_{1}, \ldots, c_{6}, t\right)=\sum_{j_{1}} \ldots \sum_{j_{6}} A_{j_{1}, \ldots, j_{6}}(t) \prod_{\kappa=1}^{6} \varphi_{j_{\kappa}}^{(\kappa)}\left(c_{\kappa}, t\right) .
$$


$\varphi_{j_{\kappa}}^{(\kappa)}\left(c_{\kappa}, t\right)$ are a total of $j_{\kappa}$ single-particle functions (SPFs) for each degree of freedom $\kappa$. $A_{\ldots j_{a}, j_{b}, \ldots}(t)=-A_{\ldots, j_{b}, j_{a}, \ldots}(t)$ and $A_{\ldots, j_{a}, j_{a}, \ldots}(t)=0$ antisymmetrize the wavefunction.

ICD is the decay of a resonance state $\Phi^{\text {res }}$ which has its three electrons in the $E_{0}, E_{0}^{\prime}$, and $A_{1}$ levels of the three respective QDs. Due to the rotational symmetry of the isolated AQD, the excited state of the central potential is two-fold degenerate. Therefore the three-QD array has two resonances as well. Block improved relaxations using the Hamiltonian of Eq. (1) without the CAP operator are used to generate the two initial states as eigenstates to that reduced Hamiltonian. Applying then the real-time equations of motion under utilization of the full Hamiltonian reveals the decay of the initial wavepacket. It is quantified through the squared autocorrelation function

$$
|a(t)|^{2}=\left|\left\langle\Phi^{r e s} \mid \Psi(t)\right\rangle\right|^{2}=e^{-\Gamma t / \hbar}
$$

i.e. the projection of the time-dependent wave function $\Psi(t)$ on the resonance function $\Phi^{\text {res }}$. Its exponential slope can be fitted to deliver the ICD rate $\Gamma$. All what was described is identical for two QDs occupied by two electrons when removing the $E^{\prime} Q D$ and the respective third electron coordinates.

This comparison of the two- and the three-electron case leads over to the relative rates for the respective processes. Although rates can be obtained numerically via electron dynamics calculations, a rationale from analytic consideration will significantly support the understanding of the underlying processes and their interrelation. Such analysis is available for two-site ICD systems in a Wigner-Weisskopf framework ${ }^{39,40}$ based on the general golden rule ansatz. This leads (under the conditions of separability of the wavefunction into a product of single-electron state functions $\phi$, no overlap of $\phi$, negligible exchange, exclusion of antisymmetrization, and deliberate assignment of certain electrons (lower index to $\phi$ ) to certain QD levels (upper index)) to a two-electron rate $^{35,39}$

$$
\Gamma^{2 e}=\left|\left\langle\phi_{1}^{A 0} \phi_{2}^{C}\left|\hat{r}_{i j}^{-1}\right| \phi_{1}^{A 1} \phi_{2}^{E 0}\right\rangle\right|^{2}
$$

Eq. (7) signifies that the single-electron states that underlie the resonance state (ket of Eq. (7)) and the multitude of final states (bra), including one electron in the continuum $C$, are Coulomb coupled in form of two independent dipole transitions of same energy in the AQD and the EQD subsystem.

The same type of derivation in the same framework of conditions has been formulated for a three-site system with three electrons. ${ }^{50}$ For the single-ionization ICD energy condition $2 \cdot I P>$ 
$\omega_{A}>1 \cdot I P$, that is subject to the present study, i.e. for the case in which one of the two EQDs is ionized during ICD, the three electron rate becomes

$$
\begin{aligned}
\Gamma^{3 e} & =\mid 2^{-1 / 2}\left\langle\phi_{2}^{A 0} \phi_{3}^{C}\left|V_{C}^{23}\right| \phi_{2}^{A 1} \phi_{3}^{E^{\prime} 0}\right\rangle\left\langle\phi_{1}^{E 0} \mid \phi_{1}^{E 0}\right\rangle \\
& +\left.2^{-1 / 2}\left\langle\phi_{1}^{C} \phi_{2}^{A 0}\left|V_{C}^{12}\right| \phi_{1}^{E 0} \phi_{2}^{A 1}\right\rangle\left\langle\phi_{3}^{E^{\prime} 0} \mid \phi_{3}^{E^{\prime} 0}\right\rangle\right|^{2} .
\end{aligned}
$$

Comparing Eqs. (7) and (8) hence reveals $\Gamma^{3 e}=2 \cdot \Gamma^{2 e}$ in agreement with the general equation

$$
\Gamma^{(N+1) e}=N \cdot \Gamma^{2 e}
$$

for $(N+1)$ electrons in $(N+1)$ subsystems indicating a linear dependence of the rate on $N$, the number of neighboring sites. ${ }^{39,40}$

\section{COMPUTATIONAL DETAILS}

\section{A. Parametrization}

The potentials defined in Eq. (2) were parametrized in a way to fulfil the energy condition for the two-electron and the single-ionization three-electron ICD process, to meet an experimentally meaningful material fabrication, and to allow for a reasonable scan over a number of inter-QD distances and angles. The computations in $\mathrm{MCTDH}$ are based on a parametrization in atomic units and such is the output. This allows comparison with the works of others on ICD in QDs. ${ }^{49,66}$ In order to make the numbers readable to QD experts as well, they are converted into materialspecific numbers for GaAs in SI units, ${ }^{45}$ by considering the dielectric constant and effective mass

of an electron in that material. ${ }^{67}$ Table I summarizes all the parameters that define the binding potentials. For the angle and distance scans, the minimal and maximal values (min, $\max$ ) are listed. Note that the parametrization with respect to the $x$ and the $z$ coordinate is identical. The potential for $R=108.3 \mathrm{~nm}$ and $\alpha=90^{\circ}$ is shown in Fig. 1 .

The MCTDH program requires further parametrization of the other operators, the DVR basis, and other technical factors. A sine DVR with 140 points was employed in a range [-541.8 a.u.;541.8 a.u.] ([-50a.u.;50a.u.]). The multi-grid potfit ${ }^{61}$ representation of the regularized Coulomb potential with $\alpha=0.1$ a.u. and $\beta=100$ a.u. is defined as in the first study on two-dimensional potentials. ${ }^{51}$ This means that the fine grid of 140 points per coordinate is supplemented by a coarse grid of only 35 points. The CAPs locate on every end of the grid starting at $c_{i}^{ \pm}= \pm 325.0 \mathrm{~nm}$ ( \pm 30 a.u.), have the order $n=4$, and the strength $\eta=8.6997 \cdot 10^{-6}$. 
TABLE I. Parametrization of the binding potentials of Eq. (2). min and max indicate ranges for parameter variations.

\begin{tabular}{ccc}
\hline \hline units & atomic & SI \\
\hline$D_{A}$ & 2.0 a.u. & $20.6 \mathrm{meV}$ \\
$b_{A}$ & 0.25 a.u. & - \\
$2 r_{A}$ & 3.33 a.u. & $36.07 \mathrm{~nm}$ \\
$D_{E}$ & 2.0 a.u. & $20.6 \mathrm{meV}$ \\
$b_{E}$ & 1.0 a.u. & - \\
$2 r_{E}$ & 1.67 a.u. & $18.03 \mathrm{~nm}$ \\
$R^{\min }$ & 10 a.u. & $108.30 \mathrm{~nm}$ \\
$R^{\max }$ & 25 a.u. & $270.75 \mathrm{~nm}$ \\
$\alpha^{\min }$ & $30^{\circ}$ & - \\
$\alpha^{\max }$ & $180^{\circ}$ & - \\
\hline \hline
\end{tabular}

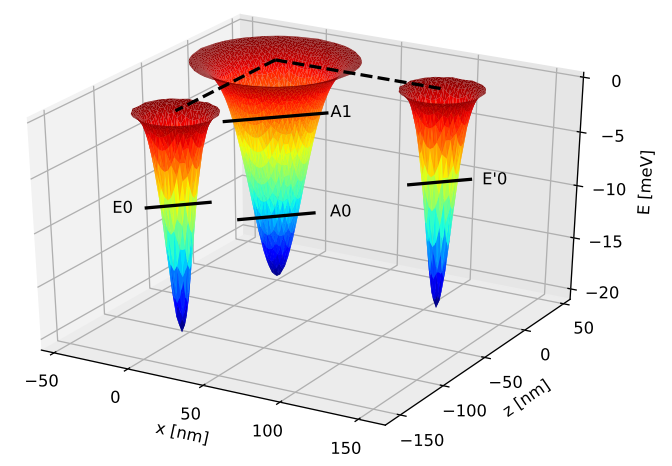

FIG. 1. Three-dimensional representation of the three two-dimensional Gaussians for $R=108.3 \mathrm{~nm}$ and $\alpha=90^{\circ}$. Horizontal black lines reflect the geometric position of the majority of electron density concerned with the indicated energetic positions of the single-electron levels $E_{0}$ and $E^{\prime}$ in each outer QDs, as well as $A_{0}$ and the two (near-)degenerate $A_{1}$ in the central QD.

MCTDH block improved relaxation calculations ${ }^{68,69}$ with $j_{\kappa}=144$ (52) SPFs (cf. Eq. (5)) in each coordinate were used to find the resonance states of two (three) electrons in two (three) QDs among the first 156 (240) states within 1.2 a.u. in time (or 0.08 ps in SI units of GaAs). The initial function for the relaxation was the antisymmetrized product of single-electron eigenfunctions representing a quartet configuration. The single-electron functions themselves were one of the 
ground state $A_{0}$ and one (two) of the lowest state on the full grid, which offer an optimal starting condition to both bound and continuum parts of the target state functions. The resonances were states no. 143 and 144 (99 and 100), respectively. They were identified by their density pattern of an s-atomic orbital-like density on the two EQDs and a p-atomic orbital-like density on the AQD (cf. Figs. 3 and 4). The thus gained resonance wavefunctions were used as initial states for the MCTDH propagations. Here only $j_{\kappa}=8 \mathrm{SPFs}$ served as functional basis. Propagations were done for 10000 a.u. in time (638.93 ps).

\section{B. Graphic Card Programming}

In order to shorten the runtimes of the computations within the MCTDH Heidelberg package ${ }^{57,58}$ in its version MCTDH84.16, usage of a graphics processing units (GPU) compilation was considered. Therefore, test computations (lower dimensional propagations and relaxations) were performed using the GNU GCC profiling tool gprof. They were analyzed based on flat profiles, i.e. summary tables listing the total time the program needs to execute each function, as well as call graphs, showing which function calls which other function how often. Based on this profiling, routines for matrix multiplications and routines for the multiplication of matrices with three-dimensional tensors were found to be particularly time-consuming. In total, these routines required up to $90 \%$ of the computing time. However, they are highly parallelizable and in particular each section is simple enough to allow for parallelization with GPUs. To this end, the respective routines were translated from Fortran77 to Fortran90 and compiler instructions for using GPUs were implemented in OpenACC. The program was compiled with the GCC9 compiler, which supports the OpenACC standard in version 2.5. MCTDH itself parallelizes superordinate routines that distribute the individual subroutines (matrix multiplications and others) onto the CPU cores. Then, one specific GPU is assigned to each of them, such that as many GPUs as CPU cores can be used.

\section{RESULTS}

\section{A. Technical Improvements: GPUs}

The modified MCTDH version is first applied to exemplary relaxation and propagation computations on three-QD three-electron ICD systems with different numbers of CPU cores and GPUs. 
Propagations showed a significant increase of computing time by a factor of up to 100 when using GPUs, irrespective of their exact number. The reason is that the multiplied matrices and tensors have so few entries (based on only eight SPFs for each degree of freedom) that the additional time needed to transfer the data from the main memory to the GPUs' memory and back clearly outweighs the gain in computation time on the GPU. Per contra, relaxation calculations use significantly more SPFs (52) which leads to much larger matrices and tensors, so that the gain in computation time on the GPUs trumps the time needed to transfer the data and a speedup is possible. Fig. 2 shows the speedup in a bar chart for different combinations of GPU and CPU cores for a block improved relaxation calculation, however, with a factor four shorter relaxation duration than what it needed in the typical calculations of this study. The increasing number of CPUs applies to different groups of bars plotted from left to right, whereas the color code marks the number of GPUs that increases within each group of bars also from left to right. The test calculations were performed on computers with two Intel Xeon Gold 6136 CPUs and up to four P100 GPUs.

While doubling the number of CPU cores from 1 to 2 and from 2 to 4 without using GPUs (blue bars) almost halves the computing time, doubling from 4 to 8 CPUs only reduces the computing time by a factor of 1.4. Any further increase of the cores (not shown in Fig. 2) does not lead to significant reductions of the computing time, meaning that the internal parallelization via $\mathrm{CPU}$ cores is exhausted with eight CPU cores. However, the additional use of GPUs for the previously mentioned routines reduces the computing time. If only comparably few compute units are GPUs (compare blue and orange bar for four and eight CPU cores), then the gain in compute time is marginal, because the relative load of the GPU is high. However, if two or four GPUs are used together with as many or more CPU cores (last two group of bars) a speedup factor of up to a 3.2 can be gained, as in the example of eight CPU cores with four GPUs compared to eight CPU cores without GPUs. This setting allows to perform the relaxation calculations needed for this study (factor four longer compared to Fig. 2) in reasonable time, i.e. within 20 hours compared to otherwise 63 hours in pure CPU parallelization and 355 hours without parallelization.

\section{B. Electronic Structure}

In the description of the ICD resonances (Sec. II) and the parametrization of the potentials (Sec. III) it was said that the central QD, the AQD, supports a ground state electronic level at energy $E_{A 0}=-11.62 \mathrm{meV}$ (-1.128 a.u.). There are further two degenerate single-electron states with 


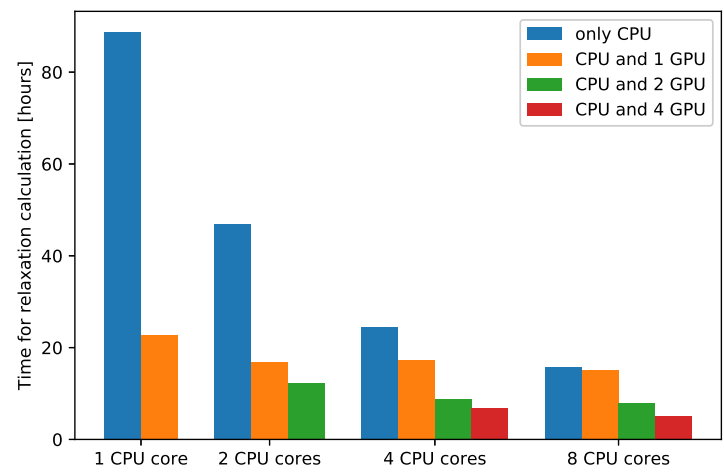

FIG. 2. Bar diagram on the compute time of a representative block-improved relaxation calculation as function of the number of CPUs (abscissa) and GPUs (color-coded bars in groups).

energy $E_{A 1}=-4.25 \mathrm{meV}$ ( -0.413 a.u.), which resemble atomic p-type orbitals perpendicular to each other. The resonant excitation energies in either direction are thus $\omega_{A}=7.37 \mathrm{meV}(0.715$ a.u.).

The two EQDs are equal in shape and support one nodal-free bound state only at energy $E_{E 0}=$ $-5.43 \mathrm{meV}$ ( -0.527 a.u.), which is the inverse to the ionization potential $I P=5.43 \mathrm{meV}$. With this energetics, the excitation energy in the central QD exceeds once the ionization energy, allowing thus for single-ionization ICD, i.e. in which only one of the EQDs is ionized. ${ }^{50}$ This choice was made, because calculations on systems that support $\omega_{A}>2 I P$ showed also only single-, but no double-ionization, ICD and were at the same time significantly less stable. ${ }^{50}$

The three-electron states are composed from the above described one-electron orbitals. Their true wavefunctions are of course influenced by the Coulomb interaction among electrons, but this does not change the fundamental level occupation underlying each of the states. The ground state has one electron in each QD in the lowest level. Therefore it is denoted $E_{0} A_{0} E_{0}^{\prime}$.

The resonances are actually the states most relevant for this work as they have a finite lifetime and decay via ICD. They have two ground state electrons in the EQDs and an excited electron in the AQD. The latter can be in one of two degenerate levels of the isolated AQD, that compare to ptype atomic orbitals. They are perpendicular to each other, but only in the linear array $\left(\alpha=180^{\circ}\right)$ they align with the $z$ and $x$ axis. In general the array's symmetry enforces the orbitals to be either parallel to the axis connecting the two EQDs or perpendicular to it, as can be rationalized by inspecting the densities in Fig. 4(a) and (b). Thus, the states are termed $E_{0} A_{1}^{\|} E_{0}^{\prime}$ and $E_{0} A_{1}^{\perp} E_{0}^{\prime}$, respectively. 


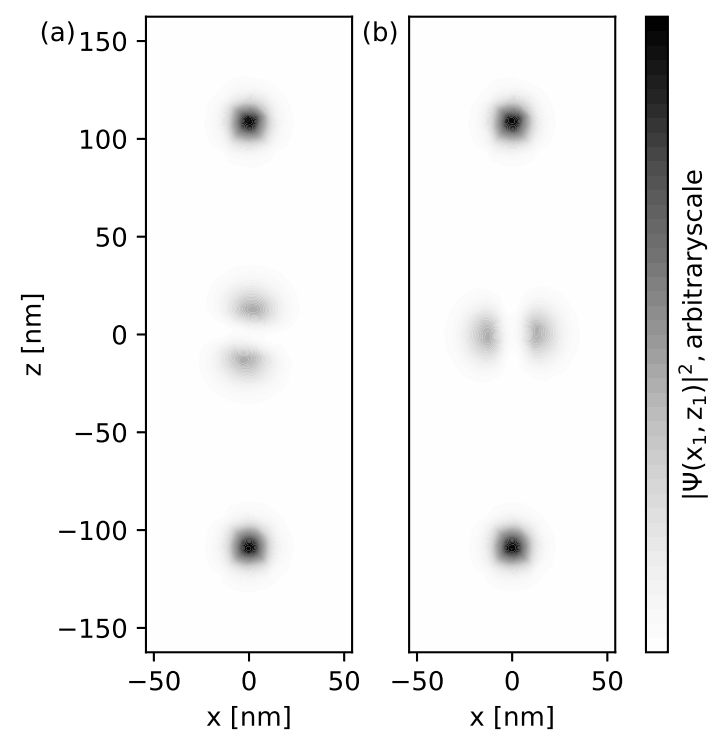

FIG. 3. Surface-projection plots of three-electron ICD resonance state densities for the linear array with $\alpha=180^{\circ}$ and $R=108.3 \mathrm{~nm}$ as function of the coordinate axes $x$ and $z$. (a) $z$-polarized resonance with $A_{1}^{\|}$, (b) $x$-polarized resonance with $A_{1}^{\perp}$.
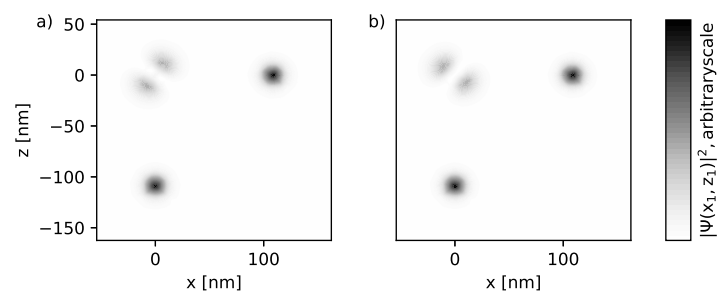

FIG. 4. Surface-projection plots of three-electron ICD resonance state densities for the angled array with $\alpha=90^{\circ}$ and $R=108.3 \mathrm{~nm}$ as function of the coordinate axes $x$ and $z$. (a) resonance with $A_{1}^{\|}$, (b) resonance with $A_{1}^{\perp}$.

Their energies depend on the exact geometric arrangement of the three QDs as is visualized in Fig. 5 for a fixed $R=108.3 \mathrm{~nm}$ and varying angles $\alpha$. It is most straightforward to analyze at first the overall trends of both curves, as both energies increase with the decrease of the angle by about $1.5 \mathrm{meV}$. This trend originates in the increasing overlap of the electrons located on the EQDs. If they are distant by $2 R=216.6 \mathrm{~nm}$ (20 a.u.) in the $\alpha=180^{\circ}$ case, they contribute by $1 / R=0.52 \mathrm{meV}(0.05$ a.u. $)$ to the total energy through their Coulomb interaction. At the high-energy side of $\alpha=30^{\circ}$, the two EQDs are as near as $56.06 \mathrm{~nm}$ (5.176 a.u.). This leads to a Coulomb energy contribution of $1 / R=1.99 \mathrm{meV}(0.193$ a.u. $)$ and ultimately explains the 


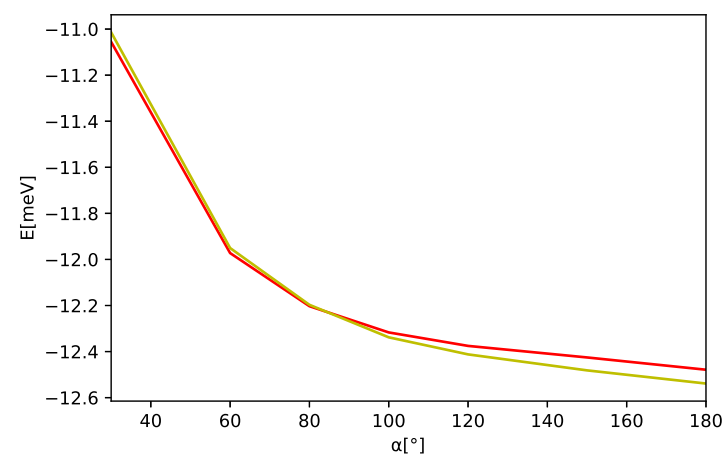

FIG. 5. Energies $E$ of the three-electron resonance states $E_{0} A_{1}^{\|} E_{0}^{\prime}$ (red/black line) and $E_{0} A_{1}^{\perp} E_{0}^{\prime}$ (yellow/gray line) as function of the angle $\alpha$ for a fixed $R=108.3 \mathrm{~nm}$.

overall increase within Fig. 5. The interesting aspect is, however, the relative energy of the parallel (red) and the perpendicular (yellow) case. In the linear array, the resonance state $E_{0} A_{1}^{\|} E_{0}^{\prime}$ has a density in which the electron on the AQD directs as much as possible towards the EQDs (Fig. 3(a)). This must lead to the highest possible orbital overlap of the electrons on the AQD and the EQDs, which is origin to the higher resonance energy displayed in Fig. 5 (red line) at $\alpha=180^{\circ}$. Contrary, the density of the perpendicular state $E_{0} A_{1}^{\perp} E_{0}^{\prime}$ (cf. Fig. 3(b)) reveals the lowest orbital overlap in a linear arrangement and thus the lower energy (yellow line). When $\alpha$ is small, the energy contribution of the interaction of electrons in the EQD and the AQD for the parallel resonance $E_{0} A_{1}^{\|} E_{0}^{\prime}$ (red line) drops compared to that as in the linear arrangement, which may be rationalized from the density representation in Fig. 4(a). Contrary, the same energy contribution in the perpendicular resonance $E_{0} A_{1}^{\perp} E_{0}^{\prime}$ increases with decreasing $\alpha$ (orange in Fig. 5; state density in Fig. 4(b)). This is the origin to the crossing of the total energy curves at $\alpha \approx 90^{\circ}$.

\section{Electron Dynamics}

\section{Linear Array}

First we investigate the distance dependence of ICD in a linearly arranged array of QDs. This is done in comparison for two and three QDs/electrons to confirm the validity of the rate prediction $\Gamma^{3 e}=2 \Gamma^{2 e}$ from the analytic Wigner-Weisskopf derivation ${ }^{39,40}$ for a system with two continuum directions. This will complement our confirmation of the rate equation for QDs in a nanowire supporting a one-dimensional continuum for the ICD electron, ${ }^{50}$ and works of others on atoms, ${ }^{39,40}$ 


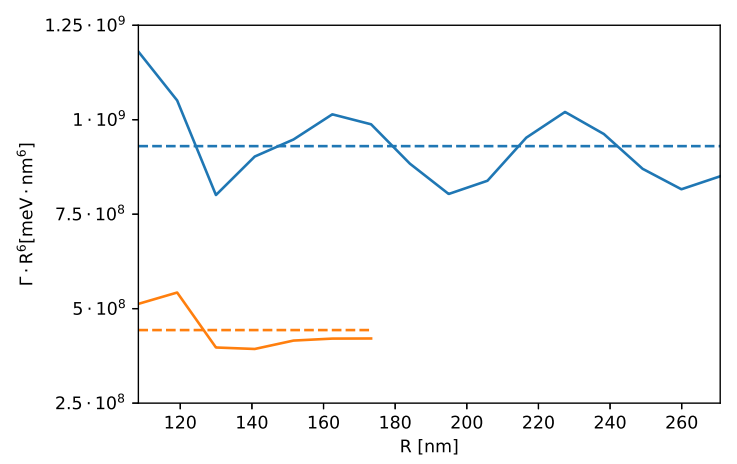

FIG. 6. $R^{6}$-normalized rates of the ICD of the $E_{0} A_{1}^{\|} E_{0}^{\prime}$ resonance in a linear QD arrangement as function of the inter-QD distance $R$. The blue (black) lines correspond to the three-QD case, the orange (gray) ones to the two-QD case. The actual values are drawn as solid lines, their averages as dashed lines.

where ICD electrons leave the system in all three spatial dimension.

As we showed in previous studies on nanowires ${ }^{35,50}$ and SA QDs, ${ }^{51}$ oscillations of the truly realized rates around the asymptote $\propto R^{-6}$ are to be expected. Hence, a sample of several $R$ is needed to evaluate the rate ratio from the averages $\Gamma^{3 e} / \Gamma^{2 e}=\overline{\Gamma^{3 e} \cdot R^{6}} / \overline{\Gamma^{2 e} \cdot R^{6}} .{ }^{50}$

Different to what was investigated in a quasi one-dimensional case, ${ }^{50}$ two resonances are available for ICD. Let us start with the z-polarized parallel resonance $\left(E_{0} A_{1}^{\|} E_{0}^{\prime}\right)$ whose density is visualized in Fig. 3(a) for three electrons. In Fig. 6 the rates for its decay multiplied by $R^{6}$ are shown as function of $R$. The blue, solid line corresponds to $\Gamma^{3 e} \cdot R$, the orange solid lines to $\Gamma^{2 e} \cdot R$. As anticipated, the rates both oscillate around their averages shown as dashed lines of same color. Note that the rates for the previously investigated $\Gamma^{2 e}$ case $^{51}$ were obtained only for a smaller span of $R$. This is because the technical parameters (cf. Sec. III) have been optimized for the new three-QD case, but lead to technical instabilities for two QDs. The maximum (average) span of oscillations is $26.9 \%(9.1 \%)$ in the three- and $22.4 \%(10.9 \%)$ in the two-electron case. This amount of oscillation is in the order of magnitude of the formerly found deviations of 50\% (24\%) for a two-dimensional continuum and thus much smaller than the respective oscillations of $222 \%$ $(85 \%)$ when only a one-dimensional continuum is available, where the ICD electron has no possibility (other than limited tunneling) to circumvent the Coulomb barrier imposed by the bound electron(s). ${ }^{51}$ The ratio is $\Gamma^{3 e} / \Gamma^{2 e}=2.10$ with a standard deviation of 0.33 . This means it supports the predicted factor 2 from the Wigner-Weisskopf theory.

The same type of analysis is made for the decay of the $x$-polarized resonance state $\left(E_{0} A_{1}^{\perp} E_{0}^{\prime}\right)$ with the density shown in Fig. 3(b). Data is to be found in Fig. 7 with the same color and 


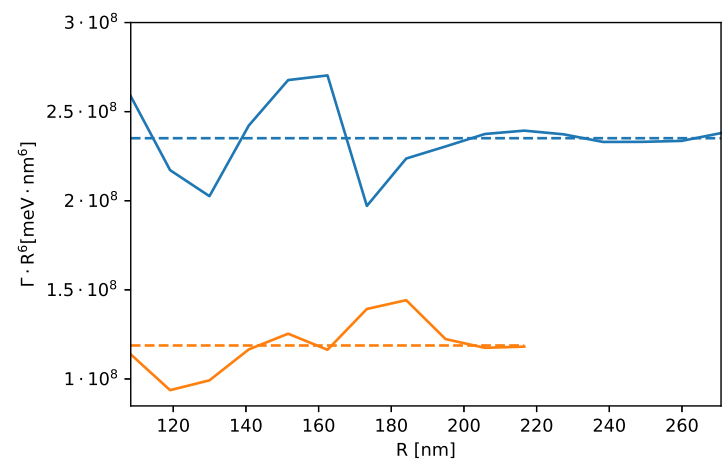

FIG. 7. $R^{6}$-normalized rates for the ICD of the $E_{0} A_{1}^{\perp} E_{0}^{\prime}$ resonance ICD in a liner QD arrangement as function of the inter-QD distance $R$. The blue (black) lines correspond to the three-QD case, the orange (gray) ones to the two-QD case. The actual values are drawn as solid lines, their averages as dashed lines.

lines-style coding as before. Again the three-electron ICD is faster by a factor of two. We obtain exactly $\Gamma^{3 e} / \Gamma^{2 e}=1.98(0.28)$, where the standard deviation is given in parentheses. This means that despite the fact that ICD rates are in general smaller for the $x$ resonance are than for the $z$ resonance, which confirms earlier results, ${ }^{51}$ the asymptotic equation keeps it generality.

\section{Angled Array}

Another feature of SA QD arrays in particular, and systems with a two-dimensional continuum in general, is the option to arrange the individual QDs in a non-linear fashion. Here we investigate the behavior of the $R^{6}$-normalized ICD rate as function of the angle $\alpha$ that determines the QD constellation. Again, the two resonances $\left(E_{0} A_{1}^{\|} E_{0}^{\prime}\right.$ and $\left.E_{0} A_{1}^{\perp} E_{0}^{\prime}\right)$ with their densities shown in Fig. 4(a) and (b), are available for the decay. Electron dynamics is only calculated exemplarily for three inter-QD distances $R=108.3,119.1,130.0 \mathrm{~nm}(10,11,12$ a.u.) for the sake of saving computer power. As $\Gamma$ is known to oscillate as function of $R$, we used the results at the three distances to average at each angle over $\Gamma_{\|}^{3 e} \cdot R^{6}$ and $\Gamma_{\perp}^{3 e} \cdot R^{6}$. Although an average over three data points is statistically not robust, one can at least deduce trends for the angle-dependence of the ICD rate.

Fig. 8 shows that $\overline{\Gamma_{\|}^{3 e} \cdot R^{6}}$ (red/black line) increases with increasing $\alpha$, while $\overline{\Gamma_{\perp}^{3 e} \cdot R^{6}}$ (yellow/gray line) contrarily decreases. This behavior is obviously caused by the overlap of the lobes of electron density that can be seen in Figs. 3 and 4, which directly connect to the Coulomb interaction as was discussed in Sec. IV B. The p-shaped density contribution on the central AQD of the parallel resonance $\left(E_{0} A_{1}^{\|} E_{0}^{\prime}\right)$ in the linear case (Fig. 3(a)) lies exactly along the array's $z$ axis. 


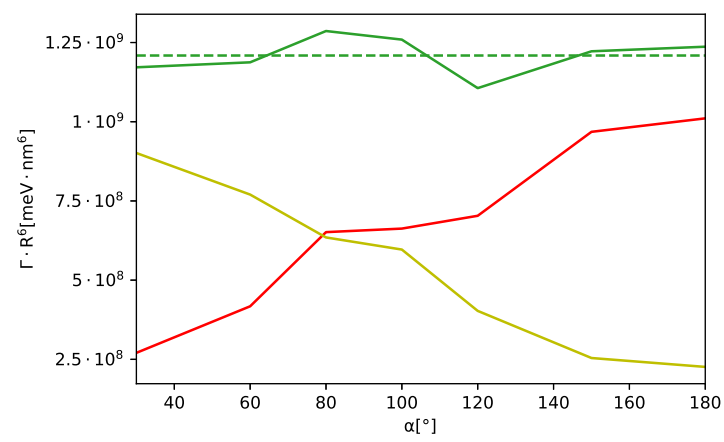

FIG. 8. $R^{6}$-normalized ICD rates as function of $\alpha . \overline{\Gamma_{\|}^{3 e} \cdot R^{6}}$ (red/black), $\overline{\Gamma_{\perp}^{3 e} \cdot R^{6}}$ (yellow/gray) and their sum (green/dark gray, top line) are displayed. The dashed line reflects the overall average rate.

This is the case of highest density overlap. When the array gets angled, then the $E_{0}$ and $E_{0}^{\prime}$ density contributions from the two emitter QDs move away from the central QD's orbital axis. Hence, the Coulomb interaction decreases and likewise the rate $\Gamma_{\|}^{3 e}$. The contrary is true for $\Gamma_{\perp}^{3 e}$. The density of an electron in the perpendicular $A_{1}^{\perp}$ level points maximally away from the densities of electrons in the two $E_{0}$ levels when $\alpha=180^{\circ}$. When the angle is small, the density contributions on the EQDs are near that lobe of the $A_{1}^{\perp}$ density which is enclosed by the angle $\alpha$. The crossing point of both curves lies near $\alpha=90^{\circ}$. The sum of both rates and its average are shown as the green (dark gray) solid and dashed lines in Fig. 8. It reflects the overall ICD rate with three electrons, which turns out to be, besides statistical fluctuations, independent of the bending angle within the array. This is an important finding in conjunction with the analytical rate equation, which states that the rate increases with the number $N$ of equidistant electron emitters irrespective of their relative arrangement. ${ }^{40}$ And indeed we find a rate ratio of $\Gamma^{3 e} / \Gamma^{2 e}=2.15(0.28)$, where for $\Gamma^{2 e}$ we use the sum of average rates obtained from Figs. 6 and 7).

In works on Ne clusters it was found that the $N$-neighbor rates would increase stronger than linearly only when the number of neighbors gets large. ${ }^{2,39}$ This was explained by the increasing overlap of electron density on the neighbors that come close together. Although in this study the two emitter QDs approach one another closely, no increase of the overall rate is observed at low angles. Either $30^{\circ}$ is not enough to reveal an enhancement effect, or the electron overlap among electrons on only two sites is not sufficient. This both supports the analytical prediction on rates increasing by factors of $N$ for small numbers of neighbors even more. ${ }^{40}$ 


\section{CONCLUSION}

The three-site interparticle Coulombic decay was investigated by means of electron dynamics calculations in a multiconfiguration time-dependent Hartree framework for the special case that a two-dimensional emission continuum is available for the ICD electron. The technical aspects of this study center around the expensive computations of this two-dimensional continuum on a large discrete variable representations grid and for three electrons. They became feasible through the recently introduced multi-grid potfit representation of the Coulomb interaction potential and an OpenACC graphic card compilation of MCTDH developed in this study. In using finally 8 CPU cores with 4 GPUs, the computing time for relaxation calculations reduces to $31.7 \%$ compared to no-GPU usage and $5.8 \%$ of what would be needed on a single CPU core. This speedup strategy make computations in even higher dimensions (more electrons or continuum dimensions) accessible. Further speedup may be achieved in coding GPU parallelization into the MCTDH code.

The physics results of this study close the knowledge gap on the three-site three-electron ICD process, which was previously only investigated both for an unconfined three-dimensional as well as a strongly-confined one-dimensional continuum for the cases of atom clusters and QDs in a nanowire, respectively. Here, self-assembled or gated laterally-arranged QDs are exemplarily studied for enabling a general understanding on ICD with a two-dimensionally unconfined electron. The findings are compared to the analytical Wigner-Weisskopf rate equation prediction in showing, as studies in the other systems, that the ICD rate is a function of the number of electron emission sites adjacent to a single excited site in their center. The result is stable for changes in the arrangement of the emitters varied through distances and angles.

\section{ACKNOWLEDGMENTS}

We gratefully acknowledge financial support from the Volkswagen Foundation through the Freigeist Fellowship No. 89525. Particular thanks goes to the HZDR offering for this work computer time on their GPU Server with 4 Tesla P100. Here we also like to thank Henrik Schulz of HZDR for his support in using the cluster. We furthermore thank Daniel Peláez for preparing the extremely useful multi-grid potfit representation of the four-dimensional Coulomb potential in a former cooperation. 


\section{AIP PUBLISHING DATA SHARING POLICY}

The data that support the findings of this study are available from the corresponding author upon reasonable request.

\section{REFERENCES}

${ }^{1}$ L. S. Cederbaum, J. Zobeley, and F. Tarantelli, Phys. Rev. Lett. 79, 4778 (1997).

${ }^{2}$ R. Santra and L. S. Cederbaum, Phys. Rep. 368, 1 (2002).

${ }^{3}$ U. Hergenhahn, J. Electron Spectrosc. Relat. Phenom. 184, 78 (2011).

${ }^{4}$ V. Averbukh, P. V. Demekhin, P. Kolorenč, S. Scheit, S. D. Stoychev, A. I. Kuleff, Y.-C. Chiang, K. Gokhberg, S. Kopelke, N. Sisourat, et al., J. Electron Spectrosc. Relat. Phenom. 183, 36 (2011).

${ }^{5}$ U. Hergenhahn, Int. J. Radiat. Biol. 88, 871 (2012).

${ }^{6}$ T. Jahnke, J. Phys. B: At. Mol. Opt. Phys. 48, 082001 (2015).

${ }^{7}$ T. Jahnke, U. Hergenhahn, B. Winter, R. Dörner, U. Frühling, P. V. Demekhin, K. Gokhberg, L. S. Cederbaum, A. Ehresmann, A. Knie, et al., Chem. Rev. 120, 11295 (2020).

${ }^{8}$ X.-J. Liu, N. Saito, H. Fukuzawa, Y. Morishita, S. Stoychev, A. Kuleff, I. H. Suzuki, Y. Tamenori, R. Richter, G. Prümper, et al., J. Phys. B: At. Mol. Opt. Phys. 40, F1 (2007).

${ }^{9}$ K. Kreidi, T. Jahnke, T. Weber, T. Havermeier, X. Liu, Y. Morishita, S. Schössler, L. Schmidt, M. Schöffler, M. Odenweller, et al., Phys. Rev. A 78, 043422 (2008).

${ }^{10}$ N. Sisourat, N. V. Kryzhevoi, P. Kolorenc, S. Scheit, T. Jahnke, and L. S. Cederbaum, Nature Phys. 6, 508 (2010).

${ }^{11}$ N. Sisourat, H. Sann, N. V. Kryzhevoi, P. Kolorenč, T. Havermeier, F. Sturm, T. Jahnke, H.-K. Kim, R. Dörner, and L. S. Cederbaum, Phys. Rev. Lett. 105, 173401 (2010).

${ }^{12}$ K. Sakai, S. Stoychev, T. Ouchi, I. Higuchi, M. Schöffler, T. Mazza, H. Fukuzawa, K. Nagaya, M. Yao, Y. Tamenori, et al., Phys. Rev. Lett. 106, 033401 (2011).

${ }^{13}$ E. Fasshauer, M. Förstel, S. Pallmann, M. Pernpointner, and U. Hergenhahn, New J. Phys. 16, 103026 (2014).

${ }^{14}$ K. Nagaya, D. Iablonskyi, N. V. Golubev, K. Matsunami, H. Fukuzawa, K. Motomura, T. Nishiyama, T. Sakai, T. Tachibana, S. Mondal, et al., Nat. Commun. 7, 13477 (2016). 
${ }^{15}$ S. Kazandjian, J. Rist, M. Weller, F. Wiegandt, D. Aslitürk, S. Grundmann, M. Kircher, G. Nalin, D. Pitters, I. Vela Pérez, et al., Phys. Rev. A 98, 050701(R) (2018).

${ }^{16}$ G. Schmid, K. Schnorr, S. Augustin, S. Meister, H. Lindenblatt, F. Trost, Y. Liu, T. Miteva, M. Gisselbrecht, S. Düsterer, et al., J. Chem. Phys. 151, 084314 (2019).

${ }^{17}$ A. Mhamdi, J. Rist, T. Havermeier, R. Dörner, T. Jahnke, and P. V. Demekhin, Phys. Rev. A 101, 023404 (2020).

${ }^{18}$ X. Ren, E. Wang, A. D. Skitnevskaya, A. B. Trofimov, K. Gokhberg, and A. Dorn, Nat. Phys. 14, 1062 (2018).

${ }^{19}$ S. Xu, D. Guo, X. Ma, X. Zhu, W. Feng, S. Yan, D. Zhao, Y. Gao, S. Zhang, X. Ren, et al., Angew. Chem. Int. Ed. 57, 17023 (2018).

${ }^{20}$ V. Averbukh and L. S. Cederbaum, Phys. Rev. Lett. 96, 053401 (2006).

${ }^{21}$ R. De, M. Magrakvelidze, M. E. Madjet, S. T. Manson, and H. S. Chakraborty, J. Phys. B 49, 11LT01 (2016).

${ }^{22}$ R. A. Wilhelm, E. Gruber, J. Schwestka, R. Kozubek, T. I. Madeira, J. P. Marques, J. Kobus, A. V. Krasheninnikov, M. Schleberger, and F. Aumayr, Phys. Rev. Lett. 119, 103401 (2017).

${ }^{23}$ B. Winter, Nucl. Instrum. Methods Phys. Res. A 601, 139 (2009).

${ }^{24}$ S. Thürmer, M. Ončák, N. Ottosson, R. Seidel, U. Hergenhahn, S. E. Bradforth, P. Slavíček, and B. Winter, Nat. Chem. 5, 590 (2013).

${ }^{25}$ K. D. Mudryk, R. Seidel, B. Winter, and I. Wilkinson, Phys. Chem. Chem. Phys. 22, 20311 (2020).

${ }^{26}$ U. Frühling, F. Trinter, F. Karimi, J. B. Williams, and T. Jahnke, J. Electron Spectrosc. Relat. Phenom. 204, 237 (2015).

${ }^{27}$ R. Santra, J. Zobeley, L. S. Cederbaum, and N. Moiseyev, Phys. Rev. Lett. 85, 4490 (2000).

${ }^{28}$ N. Moiseyev, R. Santra, J. Zobeley, and L. S. Cederbaum, J. Chem. Phys. 114, 7351 (2001).

${ }^{29}$ S. Scheit, L. S. Cederbaum, and H.-D. Meyer, J. Chem. Phys. 118, 2092 (2003).

${ }^{30}$ N. Sisourat, N. V. Kryzhevoi, P. Kolorenč, S. Scheit, and L. S. Cederbaum, Phys. Rev. A 82, 053401 (2010).

${ }^{31}$ Y.-C. Chiang, F. Otto, H.-D. Meyer, and L. S. Cederbaum, Phys. Rev. Lett. 107, 173001 (2011).

${ }^{32}$ R. Cabrera-Trujillo, O. Vendrell, and L. S. Cederbaum, Phys. Rev. A 102, 032820 (2020).

${ }^{33}$ H.-D. Meyer, U. Manthe, and L. Cederbaum, Chem. Phys. Lett. 165, 73 (1990).

${ }^{34}$ U. Manthe, H. Meyer, and L. S. Cederbaum, The Journal of Chemical Physics 97, 3199 (1992).

${ }^{35}$ A. Bande, K. Gokhberg, and L. S. Cederbaum, J. Chem. Phys. 135, 144112 (2011). 
${ }^{36}$ A. Bande, F. M. Pont, P. Dolbundalchok, K. Gokhberg, and L. S. Cederbaum, EPJ Web Conf. 41, 04031 (2013).

${ }^{37}$ T. Goldzak, L. Gantz, I. Gilary, G. Bahir, and N. Moiseyev, Phys. Rev. B 91, 165312 (2015).

${ }^{38}$ T. Goldzak, L. Gantz, I. Gilary, G. Bahir, and N. Moiseyev, Phys. Rev. B 93, 045310 (2016).

${ }^{39}$ R. Santra, J. Zobeley, and L. S. Cederbaum, Phys. Rev. B 64, 245104 (2001).

${ }^{40}$ V. Averbukh, I. B. Müller, and L. S. Cederbaum, Phys. Rev. Lett. 93, 263002 (2004).

${ }^{41}$ P. Dolbundalchok, D. Peláez, E. F. Aziz, and A. Bande, J. Comput. Chem. (2016).

${ }^{42}$ F. Weber, E. F. Aziz, and A. Bande, J. Comput. Chem. 38, 2141 (2017).

${ }^{43}$ F. M. Pont, A. Bande, and L. S. Cederbaum, Phys. Rev. B 88, 241304(R) (2013).

${ }^{44}$ A. Molle, E. Berikaa, F. M. Pont, and A. Bande, J. Chem. Phys. 150, 224105 (2019).

${ }^{45}$ A. Bande, J. Chem. Phys. 138, 214104 (2013).

${ }^{46}$ A. Haller, Y.-C. Chiang, M. Menger, E. F. Aziz, and A. Bande, Chem. Phys. 482, 135 (2017).

${ }^{47}$ A. Bande, Mol. Phys. 117, 2014 (2019).

${ }^{48}$ V. A. Guskov, F. Langkabel, M. Berg, and A. Bande, QUARKS: Braz. Electron. J. Phys. Chem. Mat. Sci., accepted (2020).

${ }^{49}$ H. Agueny, M. Pesche, B. Lutet-Toti, T. Miteva, A. Molle, J. Caillat, and N. Sisourat, Phys. Rev. B 101, 195431 (2020).

${ }^{50}$ F. Langkabel, M. Lützner, and A. Bande, J. Phys. Chem. C 123, 21757 (2019).

${ }^{51}$ A. Haller, D. Peláez, and A. Bande, J. Phys. Chem. C 123, 14754 (2019).

${ }^{52}$ P. M. Petroff, A. Lorke, and A. Imamoglu, Phys. Today 52, 46 (2001).

${ }^{53}$ L. Wang, A. Rastelli, S. Kiravittaya, M. Benyoucef, and O. G. Schmidt, Adv. Mater. 21, 2601 (2009).

${ }^{54}$ E. Zallo, P. Atkinson, L. Wang, A. Rastelli, and O. G. Schmidt, Phys. Stat. Sol. B 249, 702 (2012).

${ }^{55}$ W. G. van der Wiel, S. De Franceschi, J. M. Elzerman, T. Fujisawa, S. Tarucha, and L. P. Kouwenhoven, Rev. Mod. Phys. 75, 1 (2002).

${ }^{56}$ E. Fasshauer, M. Förstel, M. Mucke, T. Arion, and U. Hergenhahn, Chem. Phys. 482, 226 (2017).

${ }^{57}$ M. Beck, A. Jäckle, G. Worth, and H.-D. Meyer, Phys. Rep. 324, 1 (2000).

${ }^{58}$ H.-D. Meyer, F. Gatti, and G. A. Worth, eds., Multidimensional Quantum Dynamics: MCTDH Theory and Applications (Wiley-VCH, Weinheim, 2009).

${ }^{59}$ J. C. Light, Time-Dependent Quantum Molecular Dynamics (Plenum: New York, 1992), p. 185.

${ }^{60}$ J. C. Light and T. Carrington Jr, Adv. Chem. Phys. 114, 263 (2000). 
${ }^{61}$ D. Peláez and H.-D. Meyer, J. Chem. Phys. 138, 014108 (2013).

${ }^{62}$ D. Neuhauser and M. Baer, J. Chem. Phys. 90, 4351 (1989).

${ }^{63}$ D. Neuhauser and M. Baer, Comput. Phys. Comun. 63, 460 (1991).

${ }^{64}$ U. V. Riss and H.-D. Meyer, J. Phys. B 26, 4503 (1993).

${ }^{65}$ U. V. Riss and H.-D. Meyer, J. Chem. Phys. 105, 1409 (1996).

${ }^{66}$ I. Cherkes and N. Moiseyev, Phys. Rev. B 83, 113303 (2011).

${ }^{67}$ http://matprop.ru/GaAs_basic (accessed on November 21, 2018).

${ }^{68}$ H.-D. Meyer and G. A. Worth, Theor. Chem. Acc. 109, 251 (2003).

${ }^{69}$ H.-D. Meyer, F. Le Quere, C. Leonard, and F. Gatti, Chem. Phys. 329, 179 (2006). 